\title{
DYNAMIC RELIABILITY MODELING OF THREE-STATE NETWORKS
}

\author{
S. ASHRAFI, ${ }^{*}$ University of Isfahan \\ M. ASADI,** University of Isfahan and IPM
}

\begin{abstract}
This paper is an investigation into the reliability and stochastic properties of three-state networks. We consider a single-step network consisting of $n$ links and we assume that the links are subject to failure. We assume that the network can be in three states, up $(K=2)$, partial performance $(K=1)$, and down $(K=0)$. Using the concept of the two-dimensional signature, we study the residual lifetimes of the networks under different scenarios on the states and the number of failed links of the network. In the process of doing so, we define variants of the concept of the dynamic signature in a bivariate setting. Then, we obtain signature based mixture representations of the reliability of the residual lifetimes of the network states under the condition that the network is in state $K=2$ (or $K=1$ ) and exactly $k$ links in the network have failed. We prove preservation theorems showing that stochastic orderings and dependence between the elements of the dynamic signatures (which relies on the network structure) are preserved by the residual lifetimes of the states of the network (which relies on the network ageing). Various illustrative examples are also provided.
\end{abstract}

Keywords: Dynamic signature matrix; equivalent three-state network; stochastic ordering; positively quadrant dependent; association; NBU

2010 Mathematics Subject Classification: Primary 60K10

Secondary 90B25

\section{Introduction}

Most communication applications, such as rail roads, computer networks, communication channels, etc., assume the availability of a reliable network. From a mathematical point of view a network is a graph $G(V, E)$ that consists of a set of nodes (vertices), $V$, and a set of links (edges), $E$, that connect selected pairs of nodes. In a network the links or nodes are subject to failure. The failure of the links or nodes may change the state of the network. A set of special nodes are called terminals and the states of the network can be defined according to connections between terminals. In most situations, whether terminals are connected or not, the networks have several states, from perfect functioning (up state) to complete failure (down state). The networks (systems) with several states are called the multi-state networks. In this case the states of the network are usually denoted by $K=0,1, \ldots, M$, in which the state $K=0$ corresponds to the complete failure and the state $K=M$ corresponds to the complete performance of the network. Of course, the definition of the states of the network as $K=0,1,2, \ldots, M$ is not unique for a given network and the states can be defined differently by different users.

Received 9 August 2013; revision received 25 December 2013.

* Postal address: Department of Statistics, University of Isfahan, Isfahan, 81744, Iran. Email address: s.ashrafi@sci.ui.ac.ir

** Postal address: School of Mathematics, Institute of Research in Fundamental Sciences (IPM), PO Box 19395-5764, Tehran, Iran. Email address: m.asadi@sci.ui.ac.ir 
For some recent related studies on reliability properties of multi-state networks and systems, we refer the reader to [1], [10], [13], [14], [22], [24], [30], and [38].

Among the wide variety of approaches that have been employed for the evaluation of network reliability, one approach is based on the notion of the signature, as introduced in [31]. The concept of the signature is found to be a useful tool in the study of reliability and ageing properties of networks and coherent systems having two states. For recent developments on the reliability of the networks and coherent systems based on the notion of the signature, we refer the reader to, among others, [3], [5], [7]-[9], [11], [12], [15], [16], [18], [25]-[29], and [35]-[37].

This paper is an investigation on the reliability and stochastic properties of state lifetimes of single-step three-state networks based on signatures. A network is said to be single-step if the failure of one component (link or node) does not change the state of the network or changes it by one. We consider a single step three-state network consisting of $n$ links and assume that the network can be in one of three states, up $(K=2)$, partial performance $(K=1)$, and down $(K=0)$. Furthermore, we assume that the links are subject to failure and the nodes remain certainly unfailed. This paper is based on the concept of the signature for multi-state networks that was introduced in [17]. Let $X_{1}, X_{2}, \ldots, X_{n}$ be independent and identically distributed (i.i.d.) continuous random variables representing the link lifetimes of the network and denote $X_{1: n} \leq X_{2: n} \leq \cdots \leq X_{n: n}$ to be the ordered lifetimes of the links. Let the network start to function at time $t=0$ in state $K=2$. Denote $T_{1}$ and $T$ to be the times that the network enters into state $K=1$ and $K=0$, respectively. Thus, $T_{1}$ is the lifetime of the network that remains in state $K=2$ and $T$ is the network lifetime.

We should mention that $T_{1}$ and $T$ can be represented as $T_{1}=\phi_{1}\left(X_{1}, \ldots, X_{n}\right)$ and $T=$ $\phi_{2}\left(X_{1}, \ldots, X_{n}\right)$, for some functions $\phi_{1}$ and $\phi_{2}$, such that $\mathbb{P}\left(T_{1}<T\right)=1$. As an example, consider a network with four links and four nodes in which all nodes are terminals. We define the states of the network as follows: $K=2$ when all terminals are connected, $K=1$ when the network is divided into two distinct parts, and $K=0$ when the network is divided into more than two distinct parts. For this scenario, it can be seen that, $T_{1}=X_{2: 4}$, and $T=X_{3: 4}$.

The two-dimensional signature of the network is defined to be a probability matrix $S$ with elements defined by

$$
s_{i, j}=\mathbb{P}\left(T_{1}=X_{i: n}, T=X_{j: n}\right), \quad 1 \leq i<j \leq n .
$$

Denote by $S^{(1)}=\left(s_{1}^{(1)}, \ldots, s_{n}^{(1)}\right)$ and $S^{(2)}=\left(s_{1}^{(2)}, \ldots, s_{n}^{(2)}\right)$ the marginal signature vectors corresponding to the signature matrix $S$ where $s_{i}^{(1)}=\mathbb{P}\left(T_{1}=X_{i: n}\right)$ and $s_{i}^{(2)}=\mathbb{P}\left(T=X_{i: n}\right)$. The probability matrix $S$, similar to the signature vector of two-state systems, does not depend on the lifetime distribution of the links and depends only on the structure of the network. In fact, it can be shown that, for all values of $i$ and $j, s_{i, j}=n_{i, j} / n$ !, where $n_{i, j}$ is the number of ways that the failure of the $i$ th and the $j$ th links causes the states of the network to change from $K=2$ to $K=1$ and from $K=1$ to $K=0$, respectively. In fact, $s_{i, j}$ is the probability that the links with lifetimes $X_{i: n}$ and $X_{j: n}, i<j$, cause the transitions from $K=2$ to $K=1$ and from $K=1$ to $K=0$, respectively.

Let $G(x)$ and $\bar{G}(x)$ be the common distribution and survival functions of the lifetimes of the links. As the signature matrix $S$ does not depend on the distribution of $X_{i} \mathrm{~s}$, based on law of total 
probability, the joint reliability function of $\left(T_{1}, T\right)$, denoted by $\bar{G}\left(t_{1}, t\right)$, can be represented as

$$
\bar{G}\left(t_{1}, t\right)=\sum_{i=1}^{n-1} \sum_{j=i+1}^{n} s_{i, j} \mathbb{P}\left(X_{i: n}>t_{1}, X_{j: n}>t\right)
$$

where

$$
\mathbb{P}\left(X_{i: n}>t_{1}, X_{j: n}>t\right)=\sum_{k=0}^{i-1} \sum_{l=k}^{j-1} \frac{n !}{k !(l-k) !(n-l) !} G^{k}\left(t_{1}\right)\left(G(t)-G\left(t_{1}\right)\right)^{l-k} \bar{G}^{n-l}(t) ;
$$

see [17].

Samaniego et al. [32] introduced the notion of the dynamic signature and studied some ageing properties of binary systems based on this notion. In this paper we extend the notion of the dynamic signature to the bivariate setting and study several dependence and stochastic properties of residual lifetimes of the states of three-state networks based on the bivariate dynamic signature. For this purpose, we organize the paper as follows. Section 2 presents definitions and notations which are useful in the subsequent sections. Section 3 is devoted to the situations under which the network at time $t$ is in the up state and exactly $k$ links have failed. In Section 3 the notions of the dynamic signature and equivalent three-state networks are introduced and then some ageing properties of the three-state networks are explored. Some dependence and bivariate stochastic properties of the dynamic signature that are transferred to the residual lifetimes of the network states are investigated. The residual lifetimes of the network states under the condition that the network at time $t$ is in the up state are also studied. In Section 4, under the assumptions that the network at time $t$ is in state $K=1$ and exactly $k$ links have failed, we obtain the reliability function of the lifetimes of the network states. Herein, a variant of the dynamic signature is introduced. Based on this new variant the dependence between residual and inactivity lifetimes of the network states is investigated. In this section, using the definition of equivalent systems introduced by [29], several univariate stochastic ordering results are presented. Finally, the residual and inactivity lifetimes of the network states, under condition that the network at time $t$ is in state $K=1$, are studied. Various examples are also provided.

\section{Preliminaries}

In this section we provide some concepts of stochastic orders and dependence measures which will be used throughout the paper.

Definition 2.1. Let $X$ and $Y$ be two random variables with survival functions $\bar{F}(x)$ and $\bar{G}(x)$, and density functions $f(x)$ and $g(x)$, respectively.

(a) $X$ is said to be smaller than $Y$ in the usual stochastic order, denoted by $X \leq_{\text {st }} Y$, if $\bar{F}(x) \leq \bar{G}(x)$ for all $x$.

(b) $X$ is said to be smaller than $Y$ in the hazard rate order, denoted by $X \leq_{\mathrm{hr}} Y$, if $\bar{G}(x) / \bar{F}(x)$ is increasing in $x$.

(c) $X$ is said to be smaller than $Y$ in the reversed hazard rate order, denoted by $X \leq_{\mathrm{rh}} Y$, if $G(x) / F(x)$ is increasing in $x$.

(d) $X$ is said to be smaller than $Y$ in the likelihood ratio order, denoted by $X \leq_{\operatorname{lr}} Y$, if $g(x) / f(x)$ is increasing in $x$. 
(e) $X$ is said to be new better than used (NBU) if it satisfies

$$
\bar{F}(x+y) \leq \bar{F}(x) \bar{F}(y), \quad x, y>0 .
$$

Definition 2.2. Let $\boldsymbol{X}$ and $\boldsymbol{Y}$ be two random vectors with survival functions $\bar{F}$ and $\bar{G}$, respectively.

(a) $\boldsymbol{X}$ is said to be smaller than $\boldsymbol{Y}$ in the usual stochastic order (denoted by $\boldsymbol{X} \leq_{\text {st }} \boldsymbol{Y}$ ) if

$$
\mathbb{E}(\phi(\boldsymbol{X})) \leq \mathbb{E}(\phi(\boldsymbol{Y}))
$$

for every increasing function $\phi$ for which the expectations exist.

(b) $\boldsymbol{X}$ is said to be smaller than $\boldsymbol{Y}$ in the upper orthant order (denoted by $\boldsymbol{X} \leq_{\text {uo }} \boldsymbol{Y}$ ) if

$$
\bar{F}(x) \leq \bar{G}(x) \text { for all } x .
$$

For more details on various concepts of stochastic orders, we refer the reader to [33].

Definition 2.3. (a) The random variables $X$ and $Y$ are positively quadrant dependent (PQD) if, for every pair of increasing functions $h_{1}(x)$ and $h_{2}(x)$,

$$
\operatorname{cov}\left(h_{1}(X), h_{2}(Y)\right) \geq 0 .
$$

This condition is equivalent to saying that $\mathbb{P}(X>x, Y>y) \geq \mathbb{P}(X>x) \mathbb{P}(Y>y)$ for all $x$ and $y$. If the inequality sign is reversed, it is said that the random variables $X$ and $Y$ are negatively quadrant dependent (NQD).

(b) The random variables $X$ and $Y$ are associated if, for every pair of functions $h_{1}(x, y)$ and $h_{2}(x, y)$ which are increasing in each of the arguments,

$$
\operatorname{cov}\left(h_{1}(X, Y), h_{2}(X, Y)\right) \geq 0 .
$$

For more details on dependence notions, see [19] and [21].

\section{The residual reliability of the network in state $K=2$}

Consider a network consisting of $n$ links. Suppose that $X_{1}, \ldots, X_{n}$ denote the lifetimes of the links, where we assume that $X_{i}$ s are i.i.d. with a common continuous distribution function $G(x)$. Let the network be inspected at time $t$ where it is found that it is in state $K=2$ and exactly $k$ links have failed. In other words, assume that at the time of inspection the events $T_{1}>t$ and $E_{k}=\left(X_{k: n} \leq t<X_{k+1: n}\right)$ have occurred. After the failure of the $k$ th link, assume that $I_{k}$ (respectively $J_{k}$ ) denotes the number of links whose failures cause the network to enter into state $K=1$ (respectively $K=0$ ). Under this assumption, we have

$$
\mathbb{P}\left(I_{k}=i, J_{k}=j\right)=\mathbb{P}\left(T_{1}=X_{i+k: n}, T=X_{j+k: n} \mid T_{1}>t, E_{k}\right)
$$

for $i=1, \ldots, n-k$ and $j=i+1, \ldots, n-k$.

Suppose that $u=\max \left\{i \mid s_{i}^{(1)}>0\right\}$ and assume that $k \in\{0,1, \ldots, u-1\}, X_{0: n}=0$, and for such $k, \mathbb{P}\left(T_{1}>t, E_{k}\right)>0$.

The joint probability mass function of $\left(I_{k}, J_{k}\right)$, which is denoted by $S(n-k)$ is an $(n-k) \times$ $(n-k)$ matrix. In the following lemma we compute the elements of $S(n-k)$. 
Lemma 3.1. For $k \in\{0,1, \ldots, u-1\}$,

$$
\mathbb{P}\left(I_{k}=i, J_{k}=j\right)=\frac{s_{i+k, j+k}}{\bar{S}_{k}^{(1)}}, \quad i=1, \ldots, n-k, j=i+1, \ldots, n-k,
$$

where $\bar{S}_{k}^{(1)}=\sum_{i=k+1}^{n} s_{i}^{(1)}$.

Proof. First note that

$$
\mathbb{P}\left(X_{i: n}>t \mid E_{k}\right)= \begin{cases}0, & i=1, \ldots, k \\ 1, & i=k+1, \ldots, n .\end{cases}
$$

Thus, for $i=1, \ldots, n-k$ and $j=1, \ldots, n-k$, we have

$$
\begin{aligned}
\mathbb{P}\left(T_{1}\right. & \left.=X_{i+k: n}, T=X_{j+k: n} \mid T_{1}>t, E_{k}\right) \\
& =\frac{\mathbb{P}\left(T_{1}=X_{i+k: n}, T=X_{j+k: n}\right) \mathbb{P}\left(T_{1}>t, E_{k} \mid T_{1}=X_{i+k: n}, T=X_{j+k: n}\right)}{\mathbb{P}\left(T_{1}>t, E_{k}\right)} \\
& =\frac{s_{i+k, j+k} \mathbb{P}\left(E_{k}, X_{i+k: n}>t\right)}{\mathbb{P}\left(T_{1}>t, E_{k}\right)} \\
& =\frac{s_{i+k, j+k} \mathbb{P}\left(X_{i+k: n}>t \mid E_{k}\right)}{\mathbb{P}\left(T_{1}>t \mid E_{k}\right)} \\
& =\frac{s_{i+k, j+k}}{\mathbb{P}\left(T_{1}>t \mid E_{k}\right)},
\end{aligned}
$$

in which the second equality follows from the fact that the event $\left(T_{1}=X_{i: n}, T=X_{j: n}\right)$ depends only on the structure of the network (and does not depend on the lifetime distribution of the links) and the forth equality follows from (3.2). Also, using the law of total probability

$$
\begin{aligned}
\mathbb{P}\left(T_{1}>t \mid E_{k}\right) & =\sum_{i=1}^{n} \mathbb{P}\left(T_{1}=X_{i: n} \mid E_{k}\right) \mathbb{P}\left(T_{1}>t \mid E_{k}, T_{1}=X_{i: n}\right) \\
& =\sum_{i=1}^{n} s_{i}^{(1)} \mathbb{P}\left(X_{i: n}>t \mid E_{k}\right) \\
& =\sum_{i=k+1}^{n} s_{i}^{(1)},
\end{aligned}
$$

where the second equality follows from the fact that the events $\left(T_{1}=X_{i: n}\right)$ and $\left(X_{k: n} \leq t<\right.$ $\left.X_{k+1: n}\right)$ are independent.

It is interesting to note that the conditional probability (3.1), which is distribution free, can be considered as a bivariate extension of the dynamic signature introduced by [32]. We call the matrix $S(n-k)$ the dynamic signature matrix associated to the network.

In what follows, we explore the reliability and stochastic properties of the residual lifetimes of $T_{1}$ and $T$ under the condition that $\left(T_{1}>t, E_{k}\right)$. In other words, we are interested in the following conditional random variables

$$
T_{1 ; t, k}=\left(T_{1}-t \mid T_{1}>t, E_{k}\right), \quad T_{t, k}=\left(T-t \mid T_{1}>t, E_{k}\right) .
$$

The following lemma, which can be easily established by the properties of order statistics, is useful in obtaining the joint reliability function of $\left(T_{1 ; t, k}, T_{t, k}\right)$. 
Lemma 3.2. Let $X_{1}, \ldots, X_{n}$ be i.i.d. continuous random variables. For $k<i<j \leq n$, we have

$$
\left(X_{i: n}-t, X_{j: n}-t \mid X_{k: n} \leq t<X_{k+1: n}\right) \stackrel{\mathrm{D}}{=}\left(X_{i-k: n-k}^{t}, X_{j-k: n-k}^{t}\right),
$$

where ' $\stackrel{\mathrm{D}}{=}$ 'stands for equality in distribution and $X_{i-k: n-k}^{t}$ denotes the $(i-k)$ th order statistics from a random sample of size $n-k$ with survival function $\bar{G}(x \mid t)=\bar{G}(x+t) / \bar{G}(t)$ for $t, x>0$.

Lemma 3.2 leads to the following result.

$$
\begin{aligned}
\mathbb{P}\left(T_{1}-t>x_{1}, T-t>x \mid T_{1}>t, E_{k}\right) & \\
= & \sum_{i=1}^{n} \sum_{j=i+1}^{n} \mathbb{P}\left(T_{1}-t>x_{1}, T-t>x, T_{1}=X_{i: n}, T=X_{j: n} \mid T_{1}>t, E_{k}\right) \\
= & \sum_{i=k+1}^{n} \sum_{j=i+1}^{n} \mathbb{P}\left(I_{k}=i-k, J_{k}=j-k\right) \\
= & \sum_{i=k+1}^{n} \sum_{j=i+1}^{n} \mathbb{P}\left(I_{k}=i-k, J_{k}=j-k\right) \mathbb{P}\left(X_{i: n}-t>x_{1}, X_{j: n}-t>x \mid E_{k}\right) \\
= & \sum_{i=k+1}^{n} \sum_{j=i+1}^{n} \mathbb{P}\left(I_{k}=i-k, J_{k}=j-k\right) \mathbb{P}\left(X_{i-k: n-k}^{t}>x_{1}, X_{j-k: n-k}^{t}>x\right) \\
= & \sum_{i=1}^{n-k} \sum_{j=i+1}^{n-k} \mathbb{P}\left(I_{k}=i, J_{k}=j\right) \mathbb{P}\left(X_{i: n-k}^{t}>x_{1}, X_{j: n-k}^{t}>x\right) .
\end{aligned}
$$

Before stating the main results of this section, we need to introduce the notion of equivalent three-state networks, which is an extension of the notion of equivalent binary systems introduced by Navarro et al. [29]. Hence, we have the following definition.

Definition 3.1. Consider two three-state networks with i.i.d. links whose lifetimes have common distribution $G$. Let $\left(T_{1}^{(1)}, T^{(1)}\right)$ and $\left(T_{1}^{(2)}, T^{(2)}\right)$ be the corresponding lifetimes of the states. These two networks are said to be equivalent if $\left(T_{1}^{(1)}, T^{(1)}\right) \stackrel{D}{=}\left(T_{1}^{(2)}, T^{(2)}\right)$.

Let $\mathcal{A}_{i, j: m}$ be the $m \times m$ matrix such that the $(i, j)$ th element is 1 and the other elements are 0 . Also, let $\mathcal{C}_{i, j: n}, n>m$, be the $n \times n$ matrix such that its nonzero elements are defined as

$$
\begin{aligned}
c_{i+k, j+l}=\left(\begin{array}{c}
i-1+k \\
k
\end{array}\right)\left(\begin{array}{c}
j-i-1+l-k \\
l-k
\end{array}\right)\left(\begin{array}{c}
n-j-l \\
n-m-l
\end{array}\right) /\left(\begin{array}{l}
n \\
m
\end{array}\right) & \\
& k=0, \ldots, n-m, l=k, \ldots, n-m .
\end{aligned}
$$

It is clear that

$$
\mathcal{C}_{i, j: n}=\sum_{k=0}^{n-m} \sum_{l=k}^{n-m} c_{i+k, j+l} \mathcal{A}_{i+k, j+l: n}
$$


Using the above notation and Result 5.23 of [4], it can be shown that a network with $m$ i.i.d. links and signature matrix $S$ is equivalent to the network having $n$ i.i.d. links, $n>m$, with signature matrix $S^{*}$ given by

$$
S^{*}=\sum_{i=1}^{m} \sum_{j=i+1}^{m} s_{i, j} \mathcal{C}_{i, j: n}
$$

Let $S_{n}(n-k)$ be the signature matrix of order $n$ corresponding to the dynamic signature matrix $S(n-k)$, and let $\tilde{s}_{i, j}$ be the $(i, j)$ th element of $S_{n}(n-k)$.

Using the definition of equivalent networks, the reliability function of $\left(T_{1 ; t, k}, T_{t, k}\right)$ can be represented as

$$
\mathbb{P}\left(T_{1}-t>x_{1}, T-t>x \mid T_{1}>t, E_{k}\right)=\sum_{i=1}^{n} \sum_{j=i+1}^{n} \tilde{s}_{i, j} \mathbb{P}\left(X_{i: n}^{t}>x_{1}, X_{j: n}^{t}>x\right),
$$

where $X_{i: n}^{t}$ and $X_{j: n}^{t}$ were introduced in Lemma 3.2.

Samaniego et al. [32] introduced the concepts of $k$-NBU and UNBU for the binary systems. In the following definitions, we extend these notions for the three-state networks.

Definition 3.2. Consider a three-state network consisting of $n$ i.i.d. links with continuous lifetimes. Let $T_{1}$ be the lifetime of the network in state $K=2$ and $T$ be the lifetime of the network. Assume that $\left(T_{1 ; t, k}, T_{t, k}\right)$ as defined in (3.3). For fixed $k \in\{0, \ldots, u-1\}$, $\left(T_{1}, T\right)$ is said to be $k$-bivariate new better than used ( $k$-BNBU) if

$$
\left(T_{1}, T\right) \geq_{\mathrm{st}}\left(T_{1 ; t, k}, T_{t, k}\right) \quad \text { for all } t>0
$$

Definition 3.3. A three-state network is said to be uniformly bivariate new better than used (UBNBU) if $\left(T_{1}, T\right)$ is $k$-BNBU for all $k=0, \ldots, u-1$.

Using this concept, we have the following theorem.

Theorem 3.1. Consider a network with $n$ i.i.d. links $X_{1}, \ldots, X_{n}$ having a common continuous distribution function $G(x)$ and survival function $\bar{G}$. Let $S$ and $S_{n}(n-k)$ be the signature matrix and dynamic signature matrix of order $n$, respectively. If $G$ is $N B U$ and

$$
S \geq_{\text {st }} S_{n}(n-k), \quad \text { for all } k=1, \ldots, u-1,
$$

then the network is $U B N B U$.

Proof. Suppose that the residual random variable $X^{t}=\left(X_{1}-t \mid X_{1}>t\right)$ has survival function $\bar{G}(x \mid t)=\bar{G}(x+t) / \bar{G}(t)$. The condition $G$ is NBU implies that $X_{i: n} \geq_{\text {st }} X_{i: n}^{t}$ for all $i=1, \ldots, n$, where $X_{i: n}^{t}$ is the $i$ th order statistic from $\bar{G}(x \mid t)$. From Theorem 6.B.14 of [33], we can conclude that $\left(X_{i: n}, X_{j: n}\right) \geq_{\text {st }}\left(X_{i: n}^{t}, X_{j: n}^{t}\right)$ for all $1 \leq i<j \leq n$. Also, it is clear that $\left(X_{i: n}, X_{j: n}\right)$ is stochastically increasing in $(i, j)$. Hence, from representations (3.4) and (1.1) and Theorem 3.3 of [6], the assumption that $S \geq_{\mathrm{st}} S_{n}(n-k)$, for $k=1, \ldots, u-1$, gives $\left(T_{1}, T\right) \geq_{\mathrm{st}}\left(T_{1 ; t, k}, T_{t, k}\right)$ for $k=1, \ldots, u-1$. Using the same argument and the fact that, for $k=0, S_{n}(n)=S,\left(T_{1}, T\right)$ is 0-BNBU. Hence, $\left(T_{1}, T\right)$ is $k$-BNBU for all $k=0, \ldots, u-1$, that is, the network is UBNBU. 


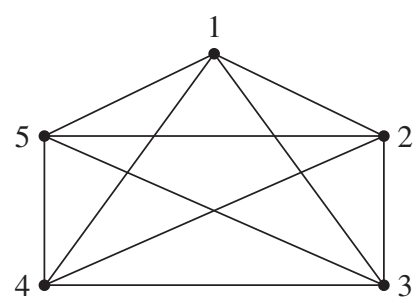

Figure 1: Network with five nodes and ten links.

Example 3.1. Figure 1 presents a network consisting of five nodes and ten links. Assume that the links are subject to failure. The states of the network are defined as $K=2$ if all nodes are connected, $K=1$ if nodes are divided into two disconnected sets, and $K=0$ if nodes are divided into at least three disconnected sets. The signature matrix of this network was estimated in [17]. The dynamic signature matrices of order $10, S_{10}(10-k)$ for $k=1, \ldots, 6$, are computed and given in Appendix A.

Assume that $\left(\tilde{I}_{1}, \tilde{J}_{1}\right)$ and $(I, J)$ have probability matrices $S_{10}(9)$ and $S$, respectively. For every increasing function $\varphi(i, j), i, j \in\{1, \ldots, 10\}$, we have

$$
\begin{aligned}
\mathbb{E}(\varphi(I, J))-\mathbb{E}\left(\varphi\left(\tilde{I}_{1}, \tilde{J}_{1}\right)\right)= & 2.38 \varphi(7,8)+0.054 \varphi(6,8)-0.1487 \varphi(6,7) \\
& -0.0003 \varphi(5,8)-0.0626 \varphi(5,7)-0.0238 \varphi(5,6) \\
& -0.0089 \varphi(4,8)-0.0231 \varphi(4,7)-0.0076 \varphi(4,6) \\
& -0.0078 \varphi(3,8)-0.0072 \varphi(3,7)-0.0019 \varphi(3,6)
\end{aligned}
$$

Thus, from the fact that $\varphi\left(i_{1}, 8\right) \geq \varphi(i, j)$ when $i_{1}=6,7, i=3, \ldots, 6, j=6,7,8$, and that the summation of the coefficients is $0, \mathbb{E}(\varphi(I, J))-\mathbb{E}\left(\varphi\left(\tilde{I}_{1}, \tilde{J}_{1}\right)\right) \geq 0$. That is, $S \geq$ st $S_{10}(9)$. Using the same argument it can be shown that $S \geq_{\text {st }} S_{10}(10-k)$ for all $k=1, \ldots, 6$. Therefore, from Theorem 3.1, if the distribution of the lifetimes of the links is NBU then the network is UBNBU.

In the sequel, we explore some dependence properties between the elements of the dynamic signature that are transferred to the lifetimes of the network states.

Theorem 3.2. Consider a network consists of $n$ i.i.d. links. Suppose that at time the network is in state $K=2$ and exactly $k$ links have failed.

(a) If $I_{k}$ and $J_{k}$ are $P Q D$ then $T_{1 ; t, k}$ and $T_{t, k}$ are $P Q D$.

(b) If $I_{k}$ and $J_{k}$ are associated then $T_{1 ; t, k}$ and $T_{t, k}$ are associated.

Proof. (a) Note that for each pair of increasing functions $\varphi(x)$ and $\psi(x)$, we have

$$
\begin{aligned}
\mathbb{E}\left(\varphi\left(T_{1 ; t, k}\right) \psi\left(T_{t, k}\right)\right) & =\sum_{i=1}^{n-k} \sum_{j=i+1}^{n-k} \mathbb{P}\left(I_{k}=i, J_{k}=j\right) \mathbb{E}\left(\varphi\left(X_{i: n-k}^{t}\right) \psi\left(X_{j: n-k}^{t}\right)\right) \\
& \geq \sum_{i=1}^{n-k} \sum_{j=i+1}^{n-k} \mathbb{P}\left(I_{k}=i, J_{k}=j\right) \mathbb{E}\left(\varphi\left(X_{i: n-k}^{t}\right)\right) \mathbb{E}\left(\psi\left(X_{j: n-k}^{t}\right)\right)
\end{aligned}
$$




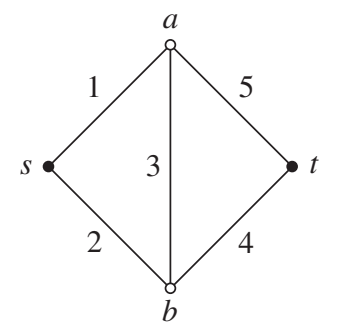

FIGURE 2: A bridge network.

$$
\begin{aligned}
\geq & \sum_{i=1}^{n-k} \sum_{j=i+1}^{n-k} \mathbb{P}\left(I_{k}=i, J_{k}=j\right) \mathbb{E}\left(\varphi\left(X_{i: n-k}^{t}\right)\right) \\
& \quad \times \sum_{i=1}^{n-k} \sum_{j=i+1}^{n-k} \mathbb{P}\left(I_{k}=i, J_{k}=j\right) \mathbb{E}\left(\psi\left(X_{j: n-k}^{t}\right)\right) \\
= & \mathbb{E}\left(\varphi\left(T_{1 ; t, k}\right)\right) \mathbb{E}\left(\psi\left(T_{t, k}\right)\right),
\end{aligned}
$$

in which the first inequality follows from the fact that $X_{i: n-k}^{t}$ and $X_{j: n-k}^{t}$ are PQD and the second inequality follows from the assumption that $I_{k}$ and $J_{k}$ are PQD and the fact that, for every increasing function $\psi, \mathbb{E}\left(\psi\left(X_{i: n-k}^{t}\right)\right)$ is increasing in $i$, completing the proof of part (a).

(b) For each pair of increasing functions $\varphi(x, y)$ and $\psi(x, y)$, we have

$$
\begin{aligned}
& \mathbb{E}\left(\varphi\left(T_{1 ; t, k}, T_{t, k}\right) \psi\left(T_{1 ; t, k}, T_{t, k}\right)\right) \\
& \quad=\sum_{i=1}^{n-k} \sum_{j=i+1}^{n-k} \mathbb{P}\left(I_{k}=i, J_{k}=j\right) \mathbb{E}\left(\varphi\left(X_{i: n-k}^{t}, X_{j: n-k}^{t}\right) \psi\left(X_{i: n-k}^{t}, X_{j: n-k}^{t}\right)\right) .
\end{aligned}
$$

Thus, the proof follows using the same steps as in the proof of part (a) and the fact that $X_{i: n-k}^{t}$ and $X_{j: n-k}^{t}$ are associated.

As applications of Theorem 3.2, we give the following examples.

Example 3.2. Consider the bridge network presented in Figure 2 which has five links, 1, 2, 3, 4,5 , and four nodes, $a, b, s, t$. Assume that links are subject to failure. We define two states for the network as follows.

(i) In the first state, we assume that the nodes $s$ and $t$ are considered as the terminals and each link has capacity one. We also assume that the states of the network are defined as the maximal flow that can be delivered from $s$ to $t$. Clearly, if all five links function or link 3 fails and other links function, the network is in state $K=2$. Also, when one of the links $1,2,4$, or 5 fails, then the flow from $s$ to $t$ reduces by one and, therefore, the state of the network is $K=1$. If there is no connection between $s$ and $t$ then $K=0$. The nonzero elements of the signature matrix of this network are given as (see Appendix B)

$$
s_{1,2}=\frac{1}{5}, \quad s_{1,3}=\frac{7}{15}, \quad s_{1,4}=\frac{2}{15}, \quad s_{2,3}=\frac{2}{15}, \quad s_{2,4}=\frac{1}{15} .
$$

We have $s_{i_{1}, j_{1}} s_{i_{2}, j_{2}} \geq s_{i_{1}, j_{2}} s_{i_{2}, j_{1}}$ for all $i_{1}<i_{2}, j_{1}<j_{2}$, which implies that $\left(I_{k}, J_{k}\right)$, $k=0,1$, is $T P_{2}$ and, hence, $I_{k}$ and $J_{k}, k=0,1$, are associated; see [19]. Therefore, from Theorem 3.2(b), $T_{1 ; t, k}$ and $T_{t, k}, k=0,1$, are associated. 
(ii) Let the nodes $s, t$, and $b$ be terminals. We assume that the network is in state $K=2$ if all terminals are connected, in state $K=1$ if two terminals are connected and in state $K=0$ if all terminals are disconnected. For instance, if links 1 and 2 fail, terminals $b$ and $t$ are connected, and in state $K=1$. In this case, it can be seen that the nonzero elements of the signature matrix of this network are given as

$$
s_{2,4}=\frac{2}{15}, \quad s_{2,5}=\frac{1}{15}, \quad s_{3,4}=\frac{7}{15}, \quad s_{3,5}=\frac{7}{30}, \quad s_{4,5}=\frac{1}{10} .
$$

It can be seen, in this case also, that, for $k=0,1,2,3, I_{k}$ and $J_{k}$ are associated and, hence, $T_{1 ; t, k}$ and $T_{t, k}$ are associated.

Example 3.3. Let us again consider Example 3.1 and assume that $k=4$. It is easy to see that $\bar{S}_{4}^{(1)}=0.9759$ and the nonzero elements of the dynamic matrix $S(6)$ are

$$
\begin{gathered}
s_{1,3}^{*}=0.0196, \quad s_{1,4}^{*}=0.0769, \\
s_{2,3}^{*}=0.0611, \quad s_{2,4}^{*}=0.2326, \quad s_{3,4}^{*}=0.6098 .
\end{gathered}
$$

This in turn implies that

$$
\sum_{l=i+1}^{n} \sum_{m=\max \{l, j\}+1}^{n} s_{l, m}^{*} \geq \sum_{l=i+1}^{n} s_{l}^{*(1)} \sum_{l=j+1}^{n} s_{l}^{*(2)}, \quad i=1,2,3, j=3,4 .
$$

Thus, $I_{4}$ and $J_{4}$ are PQD. Using the same arguments, it can be shown that, for all $k=0, \ldots, 6$, $I_{k}$ and $J_{k}$ are PQD. Therefore, $T_{1 ; t, k}$ and $T_{t, k}$ are PQD for all $k=0, \ldots, 6$.

The following theorem gives stochastic orderings of the residual lifetimes of two networks when their dynamic signatures are stochastically ordered.

Theorem 3.3. Consider two networks each consisting of $n$ links with i.i.d. lifetimes $X_{1}, \ldots, X_{n}$ and $Y_{1}, \ldots, Y_{n}$, respectively. Assume that both networks are in state $K=2$ and have exactly $k_{i}, i=1,2$, failed links at time $t$. Suppose that $S_{i, n}\left(n-k_{i}\right), i=1,2$, is the dynamic signature matrix of order $n, T_{1}^{(i)}, i=1,2$, is the lifetime in state $K=2$, and $T^{(i)}, i=1,2$, is the lifetime of the ith network. Let $T_{1 ; t, k}^{(i)}$ and $T_{t, k}^{(i)}, i=1,2$, be defined as in (3.3).

(a) If $X_{1} \leq \mathrm{hr} Y_{1}$ and $S_{1, n}\left(n-k_{1}\right) \leq_{\mathrm{uo}} S_{2, n}\left(n-k_{2}\right)$ then $\left(T_{1 ; t, k_{1}}^{(1)}, T_{t, k_{1}}^{(1)}\right) \leq_{\mathrm{uo}}\left(T_{1 ; t, k_{2}}^{(2)}, T_{t, k_{2}}^{(2)}\right)$.

(b) If $X_{1} \leq_{\mathrm{hr}} Y_{1}$ and $S_{1, n}\left(n-k_{1}\right) \leq_{\mathrm{st}} S_{2, n}\left(n-k_{2}\right)$ then $\left(T_{1 ; t, k_{1}}^{(1)}, T_{t, k_{1}}^{(1)}\right) \leq_{\mathrm{st}}\left(T_{1 ; t, k_{2}}^{(2)}, T_{t, k_{2}}^{(2)}\right)$.

Proof. Let $X_{1}^{t}=\left(X_{1}-t \mid X_{1}>t\right)$ and $Y_{1}^{t}=\left(Y_{1}-t \mid Y_{1}>t\right)$ have survival functions $\bar{G}_{1}(x \mid t)$ and $\bar{G}_{2}(x \mid t)$ and distribution functions $G_{1}(x \mid t)$ and $G_{2}(x \mid t)$, respectively. The assumption $X_{1} \leq \mathrm{hr} Y_{1}$ gives $X_{i: n}^{t} \leq_{\mathrm{st}} Y_{i: n}^{t}$ for all $i=1, \ldots, n$. Then using Theorem 6.B.14 of [33], we have $\left(X_{i: n}^{t}, X_{j: n}^{t}\right) \leq_{\mathrm{st}}\left(Y_{i: n}^{t}, Y_{j: n}^{t}\right)$ for all $1 \leq i<j \leq n$.

(a) Let $\tilde{s}_{1, i, j}$ and $\tilde{s}_{2, i, j}$ be the $(i, j)$ th elements of $S_{1, n}\left(n-k_{1}\right)$ and $S_{2, n}\left(n-k_{2}\right)$, respectively, and let $\bar{S}_{m, k, l}=\sum_{i=k+1}^{n-1} \sum_{j=\max \{i, l\}+1}^{n} \tilde{s}_{m, i, j}$ for $m=1,2$. Using representation (3.4), 
it can be seen that

$$
\begin{aligned}
\mathbb{P}\left(T_{1 ; t, k_{1}}^{(1)}>x_{1}, T_{t, k_{1}}^{(1)}>x\right) \\
=\sum_{k=0}^{n-1} \sum_{l=k}^{n} \bar{S}_{1, k, l} \frac{n !}{k !(l-k) !(n-l) !} \\
\quad \times G_{1}^{k}\left(x_{1} \mid t\right)\left(G_{1}(x \mid t)-G_{1}\left(x_{1} \mid t\right)\right)^{l-k} \bar{G}_{1}^{n-l}(x \mid t) \\
\leq \sum_{k=0}^{n-1} \sum_{l=k}^{n} \bar{S}_{2, k, l} \frac{n !}{k !(l-k) !(n-l) !} \\
\quad \times G_{1}^{k}\left(x_{1} \mid t\right)\left(G_{1}(x \mid t)-G_{1}\left(x_{1} \mid t\right)\right)^{l-k} \bar{G}_{1}^{n-l}(x \mid t) \\
\sum_{i=1}^{n-1} \sum_{j=i+1}^{n} \tilde{s}_{2, i, j} \mathbb{P}\left(X_{i: n}^{t}>x_{1}, X_{j: n}^{t}>x\right) \\
\leq \sum_{i=1}^{n-1} \sum_{j=i+1}^{n} \tilde{s}_{2, i, j} \mathbb{P}\left(Y_{i: n}^{t}>x_{1}, Y_{j: n}^{t}>x\right) \\
=\mathbb{P}\left(T_{1 ; t, k_{2}}^{(2)}>x_{1}, T_{t, k_{2}}^{(2)}>x\right),
\end{aligned}
$$

where the first inequality follows from the assumption that $S_{1, n}\left(n-k_{1}\right) \leq_{\text {uo }} S_{2, n}\left(n-k_{2}\right)$ and the second inequality follows from the fact that $\left(X_{i: n}^{t}, X_{j: n}^{t}\right) \leq_{\mathrm{st}}\left(Y_{i: n}^{t}, Y_{j: n}^{t}\right)$ implies $\left(X_{i: n}^{t}, X_{j: n}^{t}\right) \leq_{\text {uo }}\left(Y_{i: n}^{t}, Y_{j: n}^{t}\right)$.

(b) From the fact that $\left(X_{i: n}^{t}, X_{j: n}^{t}\right) \leq_{\mathrm{st}}\left(Y_{i: n}^{t}, Y_{j: n}^{t}\right)$ for all $1 \leq i<j \leq n$ and $\left(X_{i: n}^{t}, X_{j: n}^{t}\right)$ is stochastically increasing in $i, j$ then the required result follows the assumption that $S_{1, n}\left(n-k_{1}\right) \leq_{\mathrm{st}} S_{2, n}\left(n-k_{2}\right)$ and Theorem 3.3 of [6].

The following result is an immediate consequence of Theorem 3.3.

Corollary 3.1. Let $S_{n}(n-k)$ be the dynamic signature matrix of order $n$ of a network.

(a) If $S_{n}(n-k-1) \leq{ }_{\mathrm{uo}} S_{n}(n-k)$ for $k=0, \ldots, u-2$ then $\mathbb{P}\left(T_{1 ; t, k}>x_{1}, T_{t, k}>x\right)$ is decreasing in $k \in\{0, \ldots, u-1\}$.

(b) If $S_{n}(n-k-1) \leq_{\mathrm{st}} S_{n}(n-k)$ for $k=0, \ldots, u-2$ then, for every increasing function $\psi(x, y), \mathbb{E}\left(\psi\left(T_{1 ; t, k}, T_{t, k}\right)\right)$ is decreasing in $k \in\{0, \ldots, u-1\}$.

Example 3.4. Let us consider Example 3.2. For the network with the structure in part (i), suppose that at time $t$ only one link has failed. The nonzero elements of the dynamic signature matrix of order $5, S_{5}(4)$ are

$$
\tilde{s}_{1,2}=\frac{2}{5}, \quad \tilde{s}_{1,3}=\frac{4}{15}, \quad \tilde{s}_{1,4}=\frac{2}{15}, \quad \tilde{s}_{2,3}=\frac{2}{15}, \quad \tilde{s}_{2,4}=\frac{1}{15} .
$$

For the network with the structure in Example 3.2(ii), suppose that at time $t$ two links have failed. The nonzero elements of the dynamic signature matrix of order $5, S_{5}^{*}(3)$ are

$$
\begin{aligned}
& \tilde{s}_{1,2}^{*}=\frac{7}{40}, \quad \tilde{s}_{1,3}^{*}=\frac{7}{48}, \quad \tilde{s}_{1,4}^{*}=\frac{7}{60}, \quad \tilde{s}_{1,5}^{*}=\frac{7}{80}, \quad \tilde{s}_{2,3}^{*}=\frac{31}{240}, \\
& \tilde{s}_{2,4}^{*}=\frac{1}{10}, \quad \tilde{s}_{2,5}^{*}=\frac{17}{240}, \quad \tilde{s}_{3,4}^{*}=\frac{1}{12}, \quad \tilde{s}_{3,5}^{*}=\frac{13}{240}, \quad \tilde{s}_{4,5}^{*}=\frac{3}{80} \text {. }
\end{aligned}
$$


Let $\left(\tilde{I}_{k}, \tilde{J}_{k}\right)$ and $\left(\tilde{I}_{k}^{*}, \tilde{J}_{k}^{*}\right)$ have probability matrices $S_{5}(4)$ and $S_{5}^{*}(3)$, respectively. It can be seen that for every increasing function $\varphi(i, j)$,

$$
\begin{aligned}
\mathbb{E}\left(\varphi\left(\tilde{I}_{k}^{*}, \tilde{J}_{k}^{*}\right)-\varphi\left(\tilde{I}_{k}, \tilde{J}_{k}\right)\right)= & 0.225 \varphi(1,2)-0.1208 \varphi(1,3) \\
& -0.0167 \varphi(1,4)+0.0875 \varphi(1,5) \\
& -0.0042 \varphi(2,3)+0.0333 \varphi(2,4) \\
& +0.0708 \varphi(2,5)+0.0833 \varphi(3,4) \\
& +0.0542 \varphi(3,5)+0.0375 \varphi(4,5) .
\end{aligned}
$$

Using the fact that the summation of the coefficient in the above expression is 0 and $\varphi(i, j)$ is increasing in $(i, j)$, it can be seen that $\mathbb{E}\left(\varphi\left(\tilde{I}_{k}^{*}, \tilde{J}_{k}^{*}\right)\right) \geq \mathbb{E}\left(\varphi\left(\tilde{I}_{k}, \tilde{J}_{k}\right)\right)$, which implies $S_{5}^{*}(3) \geq_{\text {st }} S_{5}(4)$.

Consider the conditional random variables $T_{1, t}=\left(T_{1}-t \mid T_{1}>t\right)$ and $T_{t}=\left(T-t \mid T_{1}>\right.$ $t$ ). In what follows some dependence and stochastic properties of $\left(T_{1 ; t}, T_{t}\right)$ are explored based on properties of the dynamic signature matrix. The reliability function of $\left(T_{1 ; t}, T_{t}\right)$ can be presented as

$$
\mathbb{P}\left(T_{1}-t>x_{1}, T-t>x \mid T_{1}>t\right)=\sum_{k=0}^{u-1} p_{t}(k) \mathbb{P}\left(T_{1}-t>x_{1}, T-t>x \mid T_{1}>t, E_{k}\right)
$$

in which $p_{t}(k):=\mathbb{P}\left(E_{k} \mid T_{1}>t\right)$. Let $\phi(t)=G(t) / \bar{G}(t)$. Asadi and Berred [2] showed that

$$
p_{t}(k)=\frac{\bar{S}_{k}^{(1)}\left(\begin{array}{l}
n \\
k
\end{array}\right) \phi^{k}(t)}{\sum_{k=0}^{n-1} \bar{S}_{k}^{(1)}\left(\begin{array}{l}
n \\
k
\end{array}\right) \phi^{k}(t)} .
$$

Several properties of $p_{t}(k)$ were explored in [2].

In order to state our next results, we need the following definition (see [23]).

Definition 3.4. Suppose that at time $t$, the network is in state $K=2$. Then $\left(T_{1}, T\right)$ is said to be bivariate new better than used (BNBU) if

$$
\left(T_{1}, T\right) \geq_{\text {st }}\left(T_{1 ; t}, T_{t}\right) \text { for all } t>0 .
$$

Remark 3.1. We should point out that, if a network is UBNBU then it is also BNBU. This is because, for every increasing function $\varphi(x, y)$, we have

$$
\mathbb{E}\left(\varphi\left(T_{1 ; t}, T_{t}\right)\right)=\sum_{k=0}^{u-1} p_{t}(k) \mathbb{E}\left(\varphi\left(T_{1 ; t, k}, T_{t ; k}\right)\right) \leq \sum_{k=0}^{u-1} p_{t}(k) \mathbb{E}\left(\varphi\left(T_{1}, T\right)\right)=\mathbb{E}\left(\varphi\left(T_{1}, T\right)\right) .
$$

In the following theorem we explore dependence properties of $T_{1 ; t}$ and $T_{t}$.

Theorem 3.4. Consider a network consisting ofn i.i.d. links. Suppose that at time the network is in an up state.

(a) If $S_{n}(n-k-1) \leq_{\mathrm{uo}} S_{n}(n-k)$ for all $k=0, \ldots, u-2$ and $I_{k}$ and $J_{k}$ are $P Q D$ for all $k=0, \ldots, u-1$, then $T_{1 ; t}$ and $T_{t}$ are $P Q D$.

(b) If $S_{n}(n-k-1) \leq_{\mathrm{st}} S_{n}(n-k)$ for all $k=0, \ldots, u-2$ and $I_{k}$ and $J_{k}$ are associated for all $k=0, \ldots, u-1$, then $T_{1 ; t}$ and $T_{t}$ are associated. 
Proof. We prove part (a). The proof of part (b) is similar. Let $\varphi(x)$ and $\psi(x)$ be increasing functions. We have

$$
\begin{aligned}
\mathbb{E}\left(\varphi\left(T_{1 ; t}\right) \psi\left(T_{t}\right)\right) & =\sum_{k=0}^{u-1} p_{t}(k) \mathbb{E}\left(\varphi\left(T_{1 ; t, k}\right) \psi\left(T_{t, k}\right)\right) \\
& \geq \sum_{k=0}^{u-1} p_{t}(k) \mathbb{E}\left(\varphi\left(T_{1 ; t, k}\right)\right) \mathbb{E}\left(\psi\left(T_{t, k}\right)\right) \\
& \geq \sum_{k=0}^{u-1} p_{t}(k) \mathbb{E}\left(\varphi\left(T_{1 ; t, k}\right)\right) \sum_{k=0}^{u-1} p_{t}(k) \mathbb{E}\left(\psi\left(T_{t, k}\right)\right) \\
& =\mathbb{E}\left(\varphi\left(T_{1 ; t}\right)\right) \mathbb{E}\left(\psi\left(T_{t}\right)\right),
\end{aligned}
$$

where the first inequality follows from Theorem 3.2, in which the condition $I_{k}$ and $J_{k}$ are PQD implies that $T_{1 ; t, k}$ and $T_{t, k}$ are PQD. From Corollary 3.1, the condition $S_{n}(n-k-1) \leq_{\text {uo }}$ $S_{n}(n-k), k=0, \ldots, u-2$, gives $\mathbb{P}\left(T_{1 ; t, k}>x_{1}, T_{t, k}>x\right)$ is decreasing in $k \in\{0, \ldots, u-1\}$ and, therefore, $\mathbb{E}\left(\varphi\left(T_{1 ; t, k}\right)\right)$ and $\mathbb{E}\left(\psi\left(T_{t, k}\right)\right)$ are decreasing in $k$. Hence, the second inequality follows from the fact that every single random variable is associated.

As an application of this theorem, we present the following example.

Example 3.5. (a) Consider the network presented in Example 3.1. From Example 3.3, for $k=0, \ldots, 6, I_{k}$ and $J_{k}$ are PQD. Moreover, based on the results in Appendix A in which the dynamic signature matrices of order 10 are given, similar to Example 3.1, it can be shown that $S_{10}(10-k) \geq_{\text {st }} S_{10}(10-k-1)$ for all $k=0, \ldots, 5$, which results in $S_{10}(10-k) \geq_{\text {uo }}$ $S_{10}(10-k-1)$. Thus, from Theorem 3.4(a), $T_{1 ; t}$ and $T_{t}$ are PQD.

(b) Let us consider again Example 3.2. We have shown that, for $k=0,1, I_{k}$ and $J_{k}$ are associated. It can be seen that $S \geq_{\mathrm{st}} S_{5}(4)$. Therefore, from Theorem 3.4(b), $T_{1 ; t}$ and $T_{t}$ are associated.

In the following theorem the residual lifetimes of two networks are compared.

Theorem 3.5. Consider two networks with the same structure. Suppose that $X_{1,1}, \ldots, X_{1, n}$ and $X_{2,1}, \ldots, X_{2, n}$ are their corresponding i.i.d. lifetimes of the links, respectively. If $X_{1,1} \leq_{\mathrm{hr}}$ $X_{2,1}$ and

(a) $S_{n}(n-k-1) \leq$ uo $S_{n}(n-k)$ for all $k=0, \ldots, u-2$ then $\left(T_{1 ; t}^{(1)}, T_{t}^{(1)}\right) \leq_{\text {uo }}\left(T_{1 ; t}^{(2)}, T_{t}^{(2)}\right)$.

(b) $S_{n}(n-k-1) \leq_{\mathrm{st}} S_{n}(n-k)$ for all $k=0, \ldots, u-2$ then $\left(T_{1 ; t}^{(1)}, T_{t}^{(1)}\right) \leq_{\mathrm{st}}\left(T_{1 ; t}^{(2)}, T_{t}^{(2)}\right)$.

Proof. We present the proof for part (a). Part (b) can be proved in a similar manner. Let $X_{1,1}$ and $X_{2,1}$ have distribution functions $G_{1}(x)$ and $G_{2}(x)$, respectively, and

$$
p_{t}^{(i)}(k)=\frac{\bar{S}_{k}^{(1)}\left(\begin{array}{l}
n \\
k
\end{array}\right) \phi_{i}^{k}(t)}{\sum_{k=0}^{n-1} \bar{S}_{k}^{(1)}\left(\begin{array}{l}
n \\
k
\end{array}\right) \phi_{i}^{k}(t)}, \quad i=1,2
$$


in which $\phi_{i}(t)=G_{i}(t) / \bar{G}_{i}(t)$. Now,

$$
\begin{aligned}
\mathbb{P}\left(T_{1}^{(1)}-t>x_{1}, T^{(1)}-t>x \mid T_{1}^{(1)}>t\right) & =\sum_{k=0}^{u-1} p_{t}^{(1)}(k) \mathbb{P}\left(T_{1 ; t, k}^{(1)}>x_{1}, T_{t, k}^{(1)}>x\right) \\
& \leq \sum_{k=0}^{u-1} p_{t}^{(2)}(k) \mathbb{P}\left(T_{1 ; t, k}^{(1)}>x_{1}, T_{t, k}^{(1)}>x\right) \\
& \leq \sum_{k=0}^{u-1} p_{t}^{(2)}(k) \mathbb{P}\left(T_{1 ; t, k}^{(2)}>x_{1}, T_{t, k}^{(2)}>x\right) \\
& =\mathbb{P}\left(T_{1}^{(2)}-t>x_{1}, T^{(2)}-t>x \mid T_{1}^{(1)}>t\right),
\end{aligned}
$$

where the first inequality follows from Corollary 3.1 in which $S_{n}(n-k-1) \leq{ }_{\text {uo }} S_{n}(n-k)$, $k=0, \ldots, u-2$, gives $\mathbb{P}\left(T_{1 ; t, k}>x_{1}, T_{t, k}>x\right)$ is decreasing in $k$, and from [2, Theorem 3.4] in which $X_{1,1} \leq_{\mathrm{hr}} X_{2,1}$ gives $\sum_{k=i}^{u-1} p_{t}^{(1)}(k) \geq \sum_{k=i}^{u-1} p_{t}^{(2)}(k)$ for all $i=0, \ldots, u-1$. The second inequality follows from Theorem 3.3(a) in which $X_{1,1} \leq_{\mathrm{hr}} X_{2,1}$ gives

$$
\mathbb{P}\left(T_{1 ; t, k}^{(1)}>x_{1}, T_{t, k}^{(1)}>x\right) \leq \mathbb{P}\left(T_{1}^{(2)}>x_{1}, T^{(2)}>x\right) .
$$

\section{The reliability of the network in state $K=1$}

In this section we assume that the network is in state $K=1$ at time $t$ and exactly $k$ links have failed at that time. In other words, we consider the events $T_{1}<t<T$ and $E_{k}=\left(X_{k: n} \leq t<X_{k+1: n}\right)$. Let $\ell_{k}$ be the number of failed links before the failure of the $k$ th link that causes the network to enter into state $K=1$. Let $g_{k}$ be the number of failed links after the failure of the $k$ th link that causes the network to enter into state $K=0$. Denoting by $\delta(n-k)$ the probability matrix corresponding to the random vector $\left(\ell_{k}, g_{k}\right)$, we show that $\delta(n-k)$ is a dynamic signature. By using the same steps as in the proof of Lemma 3.1 and the fact that

$$
\mathbb{P}\left(X_{i: n}<t<X_{j: n} \mid E_{k}\right)= \begin{cases}1, & i=1, \ldots, k, j=k+1, \ldots, n, \\ 0, & \text { otherwise },\end{cases}
$$

we can show that

$$
\mathbb{P}\left(\ell_{k}=i, g_{k}=j\right)=\mathbb{P}\left(T_{1}=X_{i: n}, T=X_{j+k: n} \mid T_{1}<t<T, E_{k}\right)=\frac{s_{i, j+k}}{\xi_{k}}
$$

for $i=1, \ldots, k$ and $j=1, \ldots, n-k$, in which $\xi_{k}=\sum_{i=1}^{k} \sum_{j=k+1}^{n} s_{i, j}$. Let $S^{(1)}=$ $\left(s_{1}^{(1)}, \ldots, s_{n}^{(1)}\right)$ and $S^{(2)}=\left(s_{1}^{(2)}, \ldots, s_{n}^{(2)}\right)$ be the marginal signature vectors corresponding to the signature matrix $S$, where $s_{i}^{(1)}=\mathbb{P}\left(T_{1}=X_{i: n}\right)$ and $s_{i}^{(2)}=\mathbb{P}\left(T=X_{i: n}\right)$. It can be seen that $\xi_{k}$ can also be presented as $\xi_{k}=\sum_{i=k+1}^{n}\left(s_{i}^{(2)}-s_{i}^{(1)}\right)$. Let $l^{*}=\min \left\{i \mid s_{i}^{(1)}>0\right\}$ and $u^{*}=\max \left\{i \mid s_{i}^{(2)}>0\right\}$. Suppose that, for $k \in\left\{l^{*}, \ldots, u^{*}-1\right\}, \mathbb{P}\left(T_{1}<t<T, E_{k}\right)>0$ and $\xi_{k}>0$.

In this section we study some dependence and stochastic properties of the inactivity lifetime $\mathcal{T}_{1 ; t, k}=\left(t-T_{1} \mid T_{1}<t<T, E_{k}\right)$ and the residual lifetime $\mathcal{T}_{t, k}=\left(T-t \mid T_{1}<t<T, E_{k}\right)$. Before presenting the joint reliability function of $\left(\mathcal{T}_{1 ; t, k}, \mathcal{T}_{t, k}\right)$, we need the following lemma whose proof is a simple exercise. 
Lemma 4.1. Let $X_{1}, \ldots, X_{n}$ be i.i.d. continuous random variables from $G(x)$. Consider $X^{t}{ }_{j-k: n-k}$ as the $(j-k)$ th order statistics from a random sample of size $n-k$ from $\bar{G}(x \mid t)=$ $\bar{G}(x+t) / \bar{G}(t)$, and $X_{k-i+1: k}^{* t}$ as the $(k-i+1)$ th order statistics from a random sample of size $k$ from $G_{t}(x)=G(t-x) / G(t)$. It is easy to see that

$$
\begin{aligned}
\mathbb{P}\left(t-X_{i: n}>x_{1}, X_{j: n}-t>x \mid X_{k: n} \leq t\right. & \left.<X_{k+1: n}\right) \\
& =\mathbb{P}\left(X_{k-i+1: k}^{* t}>x_{1}\right) \mathbb{P}\left(X_{j-k: n-k}^{t}>x\right) .
\end{aligned}
$$

Based on Lemma 4.1, the joint reliability function of $\left(\mathcal{T}_{1 ; t, k}, \mathcal{T}_{t, k}\right)$ can be obtained as

$$
\begin{aligned}
\mathbb{P}\left(t-T_{1}>x_{1}, T-t>\right. & \left.x \mid T_{1}<t<T, E_{k}\right) \\
& =\sum_{i=1}^{k} \sum_{j=1}^{n-k} \mathbb{P}\left(\ell_{k}=i, g_{k}=j\right) \mathbb{P}\left(X_{k-i+1: k}^{* t}>x_{1}\right) \mathbb{P}\left(X_{j: n-k}^{t}>x\right) .
\end{aligned}
$$

It is clear that the reliability functions of $\mathcal{T}_{1 ; t, k}$ and $\mathcal{T}_{t, k}$ can, respectively, be written as

$$
\mathbb{P}\left(t-T_{1}>x_{1} \mid T_{1}<t<T, E_{k}\right)=\sum_{i=1}^{k} \mathbb{P}\left(\ell_{k}=i\right) \mathbb{P}\left(X_{k-i+1: k}^{* t}>x_{1}\right)
$$

and

$$
\mathbb{P}\left(T-t>x \mid T_{1}<t<T, E_{k}\right)=\sum_{j=1}^{n-k} \mathbb{P}\left(g_{k}=j\right) \mathbb{P}\left(X_{j: n-k}^{t}>x\right) .
$$

Let $\delta^{(1)}(n-k)$ and $f^{(2)}(n-k)$ be the marginal signature vectors respective to $\delta(n-k)$. In other words, $f^{(1)}(n-k)$ and $f^{(2)}(n-k)$ are the probability mass functions of $\ell_{k}$ and $g_{k}$, respectively. Also let $\delta_{n}^{(1)}(n-k)=\left(\tilde{s}_{1}^{(1)}, \ldots, \tilde{s}_{n}^{(1)}\right)$ and $s_{n}^{(2)}(n-k)=\left(\tilde{s}_{1}^{(2)}, \ldots, \tilde{s}_{n}^{(2)}\right)$ be the signature vectors of order $n$ corresponding to $f^{(1)}(n-k)$ and $f^{(2)}(n-k)$, respectively, which can be calculated using [29, Corollary 2.8].

Using a similar method as in [29, Corollary 2.9] and by using the equation

$$
\mathbb{P}\left(X_{k-i+1: k}>x\right)=\sum_{j=k-i+1}^{n-i+1}\left(\left(\begin{array}{l}
j-1 \\
k-i
\end{array}\right)\left(\begin{array}{l}
n-j \\
i-1
\end{array}\right) /\left(\begin{array}{l}
n \\
k
\end{array}\right)\right) \mathbb{P}\left(X_{j: n}>x\right)
$$

(see [34]), the reliability function of $\mathcal{T}_{1 ; t, k}$ can be expressed as

$$
\mathbb{P}\left(t-T_{1}>x_{1} \mid T_{1}<t<T, E_{k}\right)=\sum_{i=1}^{n} \tilde{s}_{i}^{(1)} \mathbb{P}\left(X_{n-i+1: n}^{* t}>x_{1}\right) .
$$

Also, directly from [29, Corollary 2.8], the reliability function of $\mathcal{T}_{t, k}$ can be written as

$$
\mathbb{P}\left(T-t>x \mid T_{1}<t<T, E_{k}\right)=\sum_{j=1}^{n} \tilde{s}_{j}^{(2)} \mathbb{P}\left(X_{j: n}^{t}>x\right) .
$$

In the following remark, an ageing property of the networks is investigated. 
Remark 4.1. Consider a network with $n$ i.i.d. links. From [32, Theorem 3.8], if the distribution of the lifetimes of the links is NBU and

$$
S^{(2)} \geq_{\mathrm{st}} \delta_{n}^{(2)}(n-k) \text { for } k=l^{*}, \ldots, u^{*}-1,
$$

then $T \geq_{\text {st }}\left(T-t \mid T_{1}<t<T, E_{k}\right)$ for $k=l^{*}, \ldots, u^{*}-1$; hence, from [32, Remark 3.4], $T \geq_{\text {st }}\left(T-t \mid T_{1}<t<T\right)$.

In the sequel, some dependence and stochastic properties of $\mathcal{T}_{1 ; t, k}$ and $\mathcal{T}_{t, k}$ are studied. The following theorem shows that if the dependence relationship between $\ell_{k}$ and $\mathscr{I}_{k}$ is positive then the dependence relationship between $\mathcal{T}_{1 ; t, k}$ and $\mathcal{T}_{t, k}$ is negative.

Theorem 4.1. If $\ell_{k}$ and $\mathcal{g}_{k}$ are $P Q D$ then $\mathcal{T}_{1 ; t, k}$ and $\mathcal{T}_{t, k}$ are $N Q D$. in $j$.

Proof. Note that $\mathbb{P}\left(X_{k-i+1: k}^{* t}>x_{1}\right)$ is decreasing in $i$ and $\mathbb{P}\left(X_{j-k: n-k}^{t}>x\right)$ is increasing

From the assumption that $\ell_{k}$ and $g_{k}$ are PQD, we have

$$
\begin{aligned}
\mathbb{P}\left(\mathcal{T}_{1 ; k, t}>x_{1}, \mathcal{T}_{k, t}>x\right)= & \sum_{i=1}^{k} \sum_{j=1}^{n-k} \mathbb{P}\left(\ell_{k}=i, g_{k}=j\right) \mathbb{P}\left(X_{k-i+1: k}^{* t}>x_{1}\right) \mathbb{P}\left(X_{j: n-k}^{t}>x\right) \\
\leq & \sum_{i=1}^{k} \sum_{j=1}^{n-k} \mathbb{P}\left(\ell_{k}=i, g_{k}=j\right) \mathbb{P}\left(X_{k-i+1: k}^{* t}>x_{1}\right) \\
& \times \sum_{i=1}^{k} \sum_{j=1}^{n-k} \mathbb{P}\left(\ell_{k}=i, g_{k}=j\right) \mathbb{P}\left(X_{j: n-k}^{t}>x\right) \\
= & \mathbb{P}\left(\mathcal{T}_{1 ; t, k}>x_{1}\right) \mathbb{P}\left(\mathcal{T}_{t, k}>x\right) .
\end{aligned}
$$

Hence, $\mathcal{T}_{1 ; t, k}$ and $\mathcal{T}_{t, k}$ are NQD.

Example 4.1. Let us again consider Example 3.2. For the structure of part (i) $\left(l_{k}, g_{k}\right)$ for $k=1,2,3$ is $T P_{2}$ because $s_{i_{1}, j_{1}} s_{i_{2}, j_{2}} \geq s_{i_{1}, j_{2}} s_{i_{2}, j_{1}}$ for all $i_{1}<i_{2}, j_{1}<j_{2}$. Thus, $l_{k}$ and $g_{k}$ for $k=1,2,3$ are PQD; see [19]. Therefore, for $k=1,2,3, \mathcal{T}_{1 ; t, k}$ and $\mathcal{T}_{t, k}$ are NQD. It can be seen that the same result is also true for part (ii) when $k=2,3,4$.

In the following theorem, the performance of two networks is compared based on their dynamic signature matrices.

Theorem 4.2. Consider two networks each consisting of $n$ i.i.d. links with lifetimes $X_{1}, \ldots, X_{n}$ and $Y_{1}, \ldots, Y_{n}$, respectively. Assume that both networks at time t are in state $K=1$ and have exactly $k_{1}$ and $k_{2}$ failed links, respectively. Suppose that $s_{n, i}^{(1)}\left(n-k_{1}\right)$ and $s_{n, i}^{(2)}\left(n-k_{2}\right)$, for $i=1,2$, are the marginal dynamic signature vectors of order $n$ of the ith network.

(a) If $f_{n, 1}^{(1)}\left(n-k_{1}\right) \leq_{\mathrm{st}} f_{n, 2}^{(1)}\left(n-k_{2}\right)$ and $X_{1} \leq_{\mathrm{rh}} Y_{1}$ then $\mathcal{T}_{1 ; t, k_{1}}^{(1)} \geq_{\mathrm{st}} \mathcal{T}_{1 ; t, k_{2}}^{(2)}$.

(b) If $f_{n, 1}^{(2)}\left(n-k_{1}\right) \leq_{\mathrm{st}} \delta_{n, 2}^{(2)}\left(n-k_{2}\right)$ and $X_{1} \leq_{\mathrm{hr}} Y_{1}$ then $\mathcal{T}_{t, k_{1}}^{(1)} \leq_{\mathrm{st}} \mathcal{T}_{t, k_{2}}^{(2)}$.

(c) If $f_{n, 1}^{(1)}\left(n-k_{1}\right) \leq \mathrm{hr(lr)} f_{n, 2}^{(1)}\left(n-k_{2}\right)$ and $X_{1} \stackrel{\mathrm{D}}{=} Y_{1}$ then $\mathcal{T}_{1 ; t, k_{1}}^{(1)} \geq_{\mathrm{rh}(\mathrm{lr})} \mathcal{T}_{1 ; t, k_{2}}^{(2)}$.

(d) If $f_{n, 1}^{(2)}\left(n-k_{1}\right) \leq \operatorname{hr}(\operatorname{lr}) f_{n, 2}^{(2)}\left(n-k_{2}\right)$ and $X_{1} \stackrel{\mathrm{D}}{=} Y_{1}$ then $\mathcal{T}_{t, k_{1}}^{(1)} \leq_{\mathrm{hr}(\mathrm{lr})} \mathcal{T}_{t, k_{2}}^{(2)}$.

Proof. Let $X_{1}^{* t}=\left(t-X_{1} \mid X_{1}<t\right)$ and $Y_{1}^{* t}=\left(t-Y_{1} \mid Y_{1}<t\right)$. 
(a) It can be seen that $X_{1} \leq_{\mathrm{rh}} Y_{1}$ implies $Y_{1}^{* t} \leq_{\mathrm{st}} X_{1}^{* t}$, and so $Y_{n-i+1: n}^{* t} \leq_{\mathrm{st}} X_{n-i+1: n}^{* t}$. Using the fact that $\mathbb{P}\left(Y_{n-i+1: n}^{* t}>x\right)$ is decreasing in $i$ and the assumption that $s_{n, 1}^{(1)}\left(n-k_{1}\right) \leq_{\mathrm{st}}$ $s_{n, 2}^{(1)}\left(n-k_{2}\right)$, we have

$$
\begin{aligned}
\mathbb{P}\left(\mathcal{T}_{1 ; t, k_{1}}^{(1)}>x\right) & =\sum_{i=1}^{n} \tilde{s}_{1, i}^{(1)} \mathbb{P}\left(X_{n-i+1: n}^{* t}>x\right) \\
& \geq \sum_{i=1}^{n} \tilde{s}_{1, i}^{(1)} \mathbb{P}\left(Y_{n-i+1: n}^{* t}>x\right) \\
& \geq \sum_{i=1}^{n} \tilde{s}_{2, i}^{(1)} \mathbb{P}\left(Y_{n-i+1: n}^{* t}>x\right) \\
& =\mathbb{P}\left(\mathcal{T}_{1 ; t, k_{2}}^{(2)}>x\right) .
\end{aligned}
$$

(b) The proof of part (b) is the same as part (a) and, hence, is omitted.

(c) It is well known that $X_{n-i+1: n} \geq_{\operatorname{lr}} X_{n-i: n}$. Then, for $i=1, \ldots, n$, we have

$$
X_{n-i+1: n}^{* t} \geq \operatorname{lr} X_{n-i: n}^{* t}
$$

which also implies rh-ordering. Thus, from the assumption that $f_{n, 1}^{(1)}\left(n-k_{1}\right) \leq_{\mathrm{hr}} f_{n, 2}^{(1)}\left(n-k_{2}\right)$, using [20, Theorem 2.2], we have $\mathcal{T}_{1 ; t, k_{1}}^{(1)} \geq_{\mathrm{rh}} \mathcal{T}_{1 ; t, k_{2}}^{(2)}$. The proof for lr-ordering is the same by using [20, Theorem 3.5].

(d) This part can be established in the same way as part (c) by using [33, Theorems 1.B.14 and 1.C.17] for hr- and lr-orderings, respectively.

Corollary 4.1. Let $\wp_{n}(n-k)$ be the dynamic signature matrix of order $n$ of a network.

(a) If $f_{n}^{(1)}(n-k-1) \leq_{\mathrm{st}} f_{n}^{(1)}(n-k)$ for $k=l^{*}, \ldots, u^{*}-2$ then $\mathbb{P}\left(\mathcal{T}_{1 ; t, k}>x\right)$ is increasing in $k \in\left\{l^{*}, \ldots, u^{*}-1\right\}$.

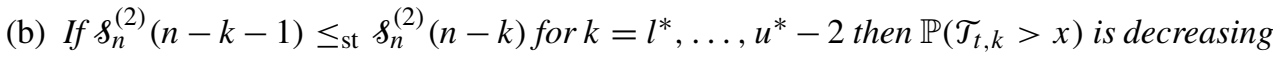
in $k \in\left\{l^{*}, \ldots, u^{*}-1\right\}$.

Example 4.2. (a) Let us consider the bridge network with the structure presented in Example 3.2(i). It is easy to see that $\xi_{1}=\frac{4}{5}, \xi_{2}=\frac{4}{5}$, and $\xi_{3}=\frac{1}{5}$. The first marginal dynamic signature vectors are

$$
f^{(1)}(4)=(1), \quad f^{(1)}(3)=\left(\frac{3}{4}, \frac{1}{4}\right), \quad f^{(1)}(2)=\left(\frac{2}{3}, \frac{1}{3}, 0\right) .
$$

The corresponding signature vectors of order 5 are given as

$$
f_{5}^{(1)}(4)=\left(\frac{1}{5}, \frac{1}{5}, \frac{1}{5}, \frac{1}{5}, \frac{1}{5}\right), \quad f_{5}^{(1)}(3)=\left(\frac{3}{10}, \frac{1}{4}, \frac{1}{5}, \frac{3}{20}, \frac{1}{10}\right), \quad f_{5}^{(1)}(2)=\left(\frac{2}{5}, \frac{3}{10}, \frac{1}{5}, \frac{1}{10}, 0\right) .
$$

It can be seen that $f_{5}^{(1)}(2) \leq_{\text {st }} f_{5}^{(1)}(3) \leq_{\text {st }} f_{5}^{(1)}(4)$.

(b) Let us consider Example 3.1. It can be seen that $\xi_{4}=0.0241, \xi_{5}=0.1183, \xi_{6}=0.4049$, and $\xi_{7}=0.9166$. The second marginal dynamic signature vectors are

$$
\begin{array}{ll}
f^{(2)}(6)=(0,0,0.195,0.805,0,0), & f^{(2)}(5)=(0,0.2012,0.7988,0,0), \\
f^{(2)}(4)=(0.206,0.794,0,0), & f^{(2)}(3)=(1,0,0) .
\end{array}
$$


The corresponding signature vectors of order 10 that are computed using MATLAB ${ }^{\circledR}$ software are given as

$$
\begin{aligned}
& f_{10}^{(2)}(6)=(0,0,0.0325,0.1132,0.2090,0.2671,0.2439,0.1342,0,0) \\
& f_{10}^{(2)}(5)=(0,0.0447,0.1225,0.1905,0.2221,0.2062,0.1474,0.0666,0,0) \\
& f_{10}^{(2)}(4)=(0.0824,0.1608,0.1931,0.1898,0.1610,0.1174,0.0690,0.0265,0,0), \\
& f_{10}^{(2)}(3)=(0.3,0.2333,0.175,0.125,0.0833,0.05,0.025,0.0083,0,0) .
\end{aligned}
$$

It can be shown that $f_{10}^{(2)}(3) \leq_{\mathrm{st}} f_{10}^{(2)}(4) \leq_{\mathrm{st}} f_{10}^{(2)}(5) \leq_{\mathrm{st}} f_{10}^{(2)}(6)$.

In the following, some dependence and stochastic properties of the conditional lifetimes $\mathcal{T}_{1 ; t}=\left(t-T_{1} \mid T_{1}<t<T\right)$ and $\mathcal{T}_{t}=\left(T-t \mid T_{1}<t<T\right)$ are studied based on properties of the dynamic signature matrix. First, we have

$$
\begin{aligned}
\mathbb{P}(t- & \left.T_{1}>x_{1}, T-t>x \mid T_{1}<t<T\right) \\
= & \sum_{k=l^{*}}^{u^{*}-1} q_{t}(k) \mathbb{P}\left(t-T_{1}>x_{1}, T-t>x \mid T_{1}<t<T, E_{k}\right)
\end{aligned}
$$

in which $q_{t}(k):=\mathbb{P}\left(E_{k} \mid T_{1}<t<T\right)$. It can be seen that

$$
q_{t}(k)=\frac{\left(\begin{array}{l}
n \\
k
\end{array}\right) \xi_{k} \phi^{k}(t)}{\sum_{r=1}^{n-1}\left(\begin{array}{l}
n \\
r
\end{array}\right) \xi_{r} \phi^{r}(t)},
$$

where $\phi(t)=G(t) / \bar{G}(t)$. It is clear that $q_{t}(k)$ represents the probability that $k$ links have failed until time $t$ when at time $t$ the network is in state $K=1$.

Using Theorem 4.1, Corollary 4.1, and the same steps as the proof of Theorem 3.4, the following theorem can be established.

Theorem 4.3. If $\ell_{k}$ and $g_{k}$ are $P Q D$ for $k=l^{*}, \ldots, u^{*}-1$ and $f_{n}^{(i)}(n-k-1) \leq_{\mathrm{st}} \ell_{n}^{(i)}(n-k)$ for $i=1,2, k=l^{*}, \ldots, u^{*}-2$, then $\mathcal{T}_{1 ; t}$ and $\mathcal{T}_{t}$ are NQD.

Remark 4.2. Asadi and Berred [2] showed that the ratio

$$
h(t)=\frac{\sum_{i=k}^{n-1}\left(\begin{array}{c}
n \\
i
\end{array}\right) \xi_{i} t^{i}}{\sum_{i=1}^{n-1}\left(\begin{array}{c}
n \\
i
\end{array}\right) \xi_{i} t^{i}}
$$

is increasing in $t$. Assume that $\phi_{i}(t)=G_{i}(t) / \bar{G}_{i}(t)$, for $i=1,2$. Since,

$$
\bar{Q}_{k}^{(i)}(t)=\sum_{j=k}^{n-1} q_{t}(j)=h\left(\phi_{i}(t)\right)
$$

for $i=1,2$, if $\bar{G}_{1}(t) \leq \bar{G}_{2}(t)$ then $\bar{Q}_{k}^{(1)}(t) \geq \bar{Q}_{k}^{(2)}(t)$.

Following the same steps as the proof of Theorem 3.5 and by using Theorem 4.2, Corollary 4.1, and Remark 4.2, we can establish the following theorem. 
Theorem 4.4. Consider two networks with the same structure. Suppose that $X_{1}, \ldots, X_{n}$ and $Y_{1}, \ldots, Y_{n}$ are their corresponding i.i.d. lifetimes of the links, respectively.
(a) If $f_{n}^{(1)}(n-k-1) \leq_{\mathrm{st}} \delta_{n}^{(1)}(n-k)$ for $k=l^{*}, \ldots, u^{*}-2$ and $X_{1} \leq_{\mathrm{rh}} Y_{1}$ then $\mathcal{T}_{1 ; t}^{(1)} \geq_{\mathrm{st}} \mathcal{T}_{1 ; t}^{(2)}$.

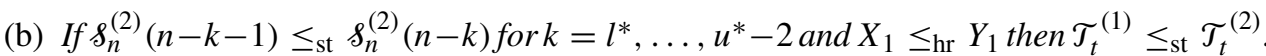

\section{Conclusions}

In this paper we considered a three-state network with states $K=0,1,2$, where $K=2$ corresponds to the perfect performance of the network, $K=1$ shows the partial performance, and $K=0$ corresponds to failure of the network. We have considered situations under which the network at time $t$ is in the up state $(K=2)$ and exactly $k$ links have failed. Under this condition, we defined the notion of the dynamic signature and explored some ageing properties of the networks. Dependence and bivariate stochastic properties of the dynamic signature that are transferred to the residual lifetimes of the network states have been investigated. Then, under the assumptions that the network at time $t$ is in state $K=1$ and exactly $k$ links have failed, and using a variant of the dynamic signature, we obtained a reliability function of the residual and the inactivity lifetimes of the network states. The dependence relationship between residual and inactivity lifetimes of the network states have been explored. Finally, based on the definition of equivalent systems, introduced in [29], several univariate stochastic ordering results were presented. An interesting problem which can be considered for future study is to extend the results of this paper to networks with more than three states.

\section{Appendix A. Computation of the signature and dynamic signature in Example 3.1}

The nonzero elements of the signature matrix $S$ of Example 3.1, are estimated by [17] as follows.

$$
\begin{aligned}
& s_{4,7}=0.0047, \quad s_{4,8}=0.0194, \quad s_{5,7}=0.0191, \quad s_{5,8}=0.0751, \\
& s_{6,7}=0.0596, \quad s_{6,8}=0.227, \quad s_{7,8}=0.5951 .
\end{aligned}
$$

From this, the dynamic signature matrices of order $10, \varsigma_{10}(10-k)$ can be calculated using MATLAB ${ }^{\circledR}$ software as follows:

(I) The nonzero elements of $\varsigma_{10}(9)$ are

$$
\begin{aligned}
& \tilde{s}_{3,6}=0.0019, \quad \tilde{s}_{3,7}=0.0072, \quad \tilde{s}_{3,8}=0.0078, \quad \tilde{s}_{4,6}=0.0076 \text {, } \\
& \tilde{s}_{4,7}=0.0278, \quad \tilde{s}_{4,8}=0.0284, \quad \tilde{s}_{5,6}=0.0238, \quad \tilde{s}_{5,7}=0.0817 \text {, } \\
& \tilde{s}_{5,8}=0.0754, \quad \tilde{s}_{6,7}=0.2083, \quad \tilde{s}_{6,7}=0.1730, \quad \tilde{s}_{7,8}=0.3571 \text {. }
\end{aligned}
$$

(II) The nonzero elements of $\varsigma_{10}(8)$ are

$$
\begin{array}{lllll}
\tilde{s}_{2,5}=0.0010, & \tilde{s}_{2,6}=0.0038, & \tilde{s}_{2,7}=0.0058, & \tilde{s}_{2,8}=0.0043, & \tilde{s}_{3,5}=0.0042, \\
\tilde{s}_{3,6}=0.0142, & \tilde{s}_{3,7}=0.0195, & \tilde{s}_{3,8}=0.0135, & \tilde{s}_{4,5}=0.0132, & \tilde{s}_{4,6}=0.0407, \\
\tilde{s}_{4,7}=0.0495, & \tilde{s}_{4,8}=0.0314, & \tilde{s}_{5,6}=0.1005, & \tilde{s}_{5,7}=0.1081, & \tilde{s}_{5,8}=0.0636, \\
\tilde{s}_{6,7}=0.2116, & \tilde{s}_{6,8}=0.1166, & \tilde{s}_{7,8}=0.1984 .
\end{array}
$$


(III) The nonzero elements of $\$_{10}(7)$ are

$$
\begin{array}{lllll}
\tilde{s}_{1,4}=0.0008, & \tilde{s}_{1,5}=0.0028, & \tilde{s}_{1,6}=0.0048, & \tilde{s}_{1,7}=0.0052, & \tilde{s}_{1,8}=0.0032, \\
\tilde{s}_{2,4}=0.0032, & \tilde{s}_{2,5}=0.0098, & \tilde{s}_{2,6}=0.0146, & \tilde{s}_{2,7}=0.0141, & \tilde{s}_{2,8}=0.0079, \\
\tilde{s}_{3,4}=0.0099, & \tilde{s}_{3,5}=0.0271, & \tilde{s}_{3,6}=0.0349, & \tilde{s}_{3,7}=0.0303, & \tilde{s}_{3,8}=0.0157, \\
\tilde{s}_{4,5}=0.0645, & \tilde{s}_{4,6}=0.0717, & \tilde{s}_{4,7}=0.0570, & \tilde{s}_{4,8}=0.0278, & \tilde{s}_{5,6}=0.1309, \\
\tilde{s}_{5,7}=0.0972, & \tilde{s}_{5,8}=0.0450, & \tilde{s}_{6,7}=0.1537, & \tilde{s}_{6,8}=0.0685, & \tilde{s}_{7,8}=0.0992 .
\end{array}
$$

(IV) The nonzero elements of $\varsigma_{10}(6)$ are

$$
\begin{array}{lllll}
\tilde{s}_{1,3}=0.0033, & \tilde{s}_{1,4}=0.0092, & \tilde{s}_{1,5}=0.0138, & \tilde{s}_{1,6}=0.0147, & \tilde{s}_{1,7}=0.0115, \\
\tilde{s}_{1,8}=0.0055, & \tilde{s}_{2,3}=0.0102, & \tilde{s}_{2,4}=0.0243, & \tilde{s}_{2,5}=0.0306, & \tilde{s}_{2,6}=0.0288, \\
\tilde{s}_{2,7}=0.0206, & \tilde{s}_{2,8}=0.0092, & \tilde{s}_{3,4}=0.0552, & \tilde{s}_{3,5}=0.0579, & \tilde{s}_{3,6}=0.0493, \\
\tilde{s}_{3,7}=0.0328, & \tilde{s}_{3,8}=0.0140, & \tilde{s}_{4,5}=0.0958, & \tilde{s}_{4,6}=0.0761, & \tilde{s}_{4,7}=0.0482, \\
\tilde{s}_{4,8}=0.0198, & \tilde{s}_{5,6}=0.1092, & \tilde{s}_{5,7}=0.0668, & \tilde{s}_{5,8}=0.0267, & \tilde{s}_{6,7}=0.0886, \\
\tilde{s}_{6,8}=0.0346, & \tilde{s}_{7,8}=0.0436 .
\end{array}
$$

(V) The nonzero elements of $\wp_{10}(5)$ are

$$
\begin{array}{lllll}
\tilde{s}_{1,2}=0.0150, & \tilde{s}_{1,3}=0.0308, & \tilde{s}_{1,4}=0.0360, & \tilde{s}_{1,5}=0.0333, & \tilde{s}_{1,6}=0.0256, \\
\tilde{s}_{1,7}=0.0156, & \tilde{s}_{1,8}=0.0061, & \tilde{s}_{2,3}=0.0656, & \tilde{s}_{2,4}=0.0609, & \tilde{s}_{2,5}=0.0499, \\
\tilde{s}_{2,6}=0.0355, & \tilde{s}_{2,7}=0.0206, & \tilde{s}_{2,8}=0.0078, & \tilde{s}_{3,4}=0.0857, & \tilde{s}_{3,5}=0.0665, \\
\tilde{s}_{3,6}=0.0455, & \tilde{s}_{3,7}=0.0255, & \tilde{s}_{3,8}=0.0094, & \tilde{s}_{4,5}=0.0830, & \tilde{s}_{4,6}=0.0554, \\
\tilde{s}_{4,7}=0.0305, & \tilde{s}_{4,8}=0.0111, & \tilde{s}_{5,6}=0.0654, & \tilde{s}_{5,7}=0.0355, & \tilde{s}_{5,8}=0.0128, \\
\tilde{s}_{6,7}=0.0404, & \tilde{s}_{6,8}=0.0144, & \tilde{s}_{7,8}=0.0161 .
\end{array}
$$

(VI) The nonzero elements of $\varsigma_{10}(4)$ are

$$
\begin{array}{lllll}
\tilde{s}_{1,2}=0.1333, & \tilde{s}_{1,3}=0.1000, & \tilde{s}_{1,4}=0.0714, & \tilde{s}_{1,5}=0.0476, & \tilde{s}_{1,6}=0.0286, \\
\tilde{s}_{1,7}=0.0143, & \tilde{s}_{1,8}=0.0048, & \tilde{s}_{2,3}=0.1000, & \tilde{s}_{2,4}=0.0714, & \tilde{s}_{2,5}=0.0476, \\
\tilde{s}_{2,6}=0.0286, & \tilde{s}_{2,7}=0.0143, & \tilde{s}_{2,8}=0.0048, & \tilde{s}_{3,4}=0.0714, & \tilde{s}_{3,5}=0.0476, \\
\tilde{s}_{3,6}=0.0286, & \tilde{s}_{3,7}=0.0143, & \tilde{s}_{3,8}=0.0048, & \tilde{s}_{4,5}=0.0476, & \tilde{s}_{4,6}=0.0286, \\
\tilde{s}_{4,7}=0.0143, & \tilde{s}_{4,8}=0.0048, & \tilde{s}_{5,6}=0.0286, & \tilde{s}_{5,7}=0.0143, & \tilde{s}_{5,8}=0.0048, \\
\tilde{s}_{6,7}=0.0143, & \tilde{s}_{6,8}=0.0048, & \tilde{s}_{7,8}=0.0048 .
\end{array}
$$

\section{Appendix B. Computation of the signature in Example 3.2}

We show the computation regarding part (i). The number of possible orderings of the link failures is $5 !=120$. We can easily verify that in 24 situations out of 120 , the first and second failure links cause the states of the network change from $K=2$ to $K=1$ and $K=1$ to $K=0$, respectively; that is, $n_{1,2}=24$. Similarly, it can be seen that $n_{1,3}=56, n_{1,4}=16, n_{2,3}=16$, $n_{2,4}=8$. Hence, based on the fact that $s_{i, j}=n_{i, j} / n$ !, the nonzero elements of the signature matrix of this network are given, respectively, as $s_{1,2}=\frac{24}{120}, s_{1,3}=\frac{56}{120}, s_{1,4}=\frac{16}{120}, s_{2,3}=\frac{16}{120}$, $s_{2,4}=\frac{8}{120}$.

The computation for part (ii) can be achieved in a similar way; hence, we omit the details. 


\section{Acknowledgements}

We would like to express our sincere thanks to an associate editor and an anonymous referee for their constructive comments and suggestions which improved the presentation of this paper. M. Asadi's research was carried out in IPM Isfahan branch and was in part supported by a grant from IPM (grant number 92620411).

\section{References}

[1] Amari, S. V., Zuo, M. J. And Dill, G. (2009). A fast and robust reliability evaluation algorithm for generalized multi-state $k$-out-of- $n$ systems. IEEE Trans. Reliab. 58, 88-97.

[2] Asadi, M. and Berred, A. (2012). On the number of failed components in a coherent operating system. Statist. Prob. Lett. 82, 2156-2163.

[3] Asadi, M. and Goliforushani, S. (2008). On the mean residual life function of coherent systems. IEEE Trans. Reliab. 57, 574-580.

[4] Balakrishnan, N. (2007). Permanents, order statistics, outliers, and robustness. Rev. Mat. Complut. 20, 7-107.

[5] Balakrishnan, N. AND Asadi, M. (2012). A proposed measure of residual life of live components of a coherent system. IEEE Trans. Reliab. 61, 41-49.

[6] Belzunce, F., Mercader, J.-A., Ruiz, J.-M. and Spizzichino, F. (2009). Stochastic comparisons of multivariate mixture models. J. Multivariate Anal. 100, 1657-1669.

[7] Boland, P. J., Samaniego, F. J. And Vestrup, E. M. (2003). Linking dominations and signatures in network reliability theory. In Mathematical and Statistical Methods in Reliability, World Scientific, River Edge, NJ, pp. 89-103.

[8] DA, G. AND Hu, T. (2013). On bivariate signatures for systems with independent modules. In Stochastic Orders in Reliability and Risk, Springer, New York, pp. 143-166.

[9] Eryilmaz, S. (2010). Conditional lifetimes of consecutive $k$-out-of- $n$ systems. IEEE Trans. Reliab. 59, $178-182$.

[10] Eryilmaz, S. (2010). Mean residual and mean past lifetime of multi-state systems with identical components. IEEE Trans. Reliab. 59, 644-649.

[11] Eryilmaz, S. (2010). Mixture representations for the reliability of consecutive- $k$ systems. Math. Comput. Modelling 51, 405-412.

[12] Eryilmaz, S. (2010). Number of working components in consecutive $k$-out-of- $n$ system while it is working. Commun. Statist. Simul. Comput. 39, 683-692.

[13] Eryilmaz, S. (2011). Dynamic reliability and performance evaluation of multi-state systems with two components. Hacet. J. Math. Statist. 40, 125-133.

[14] ERYIlmaz, S. AND İşÇıOĞLU, F. (2011). Reliability evaluation for a multi-state system under stress-strength setup. Commun. Statist. Theory Meth. 40, 547-558.

[15] Eryilmaz, S. ANd Zuo, M. J. (2010). Computing and applying the signature of a system with two common failure criteria. IEEE Trans. Reliab. 59, 576-580.

[16] Feng, X., Zhang, S. And Li, X. (2013). A note on the mixture representation of the conditional residual lifetime of a coherent system. J. Appl. Prob. 50, 475-485.

[17] Gertsbakh, I. B. ANd ShPUngIn, Y. (2012). Stochastic models of network survivability. QTQM 9, 45-58.

[18] Goliforushani, S., Asadi, M. and Balakrishnan, N. (2012). On the residual and inactivity times of the components of used coherent systems. J. Appl. Prob. 49, 385-404.

[19] Joe, H. (1997). Multivariate Models and Dependence Concepts. Chapman and Hall, London.

[20] Khaledi, B.-E. AND SHaKed, M. (2010). Stochastic comparisons of multivariate mixtures. J. Multivariate Anal. 101, 2486-2498.

[21] LaI, C.-D. AND XIE, M. (2006). Stochastic Ageing and Dependence for Reliability. Springer, New York.

[22] LI, W. AND ZUo, M. J. (2008). Reliability evaluation of multi-state weighted $k$-out-of- $n$ systems. Reliab. Eng. Syst. Saf. 93, 160-167.

[23] Li, X. and Pellerey, F. (2011). Generalized Marshall-Olkin distributions and related bivariate aging properties. J. Multivariate Anal. 102, 1399-1409.

[24] Lisnianski, A. and Levitin, G. (2003). Multi-State System Reliability: Assessment, Optimization and Applications. World Scientific, River Edge, NJ.

[25] NAVARro, J. AND Rychlik, T. (2007). Reliability and expectation bounds for coherent systems with exchangeable components. J. Multivariate Anal. 98, 102-113.

[26] Navarro, J., Balakrishnan, N. and Samaniego, F. J. (2008). Mixture representations of residual lifetimes of used systems. J. Appl. Prob. 45, 1097-1112.

[27] Navarro, J., Samaniego, F. J. and Balakrishnan, N. (2010). The joint signature of coherent systems with shared components. J. Appl. Prob. 47, 235-253. 
[28] Navarro, J., Samaniego, F. J. and Balakrishnan, N. (2011). Signature-based representations for the reliability of systems with heterogeneous components. J. Appl. Prob. 48, 856-867.

[29] Navarro, J., Samaniego, F. J., Balakrishnan, N. and Bhattacharya, D. (2008). On the application and extension of system signatures in engineering reliability. Naval Res. Logistics $\mathbf{5 5}, 313-327$.

[30] Ramirez-Marquez, J. E. and Coit, D. W. (2005). A Monte-Carlo simulation approach for approximating multi-state two-terminal reliability. Reliab. Eng. Syst. Saf. 87, 253-264.

[31] Samaniego, F. J. (1985). On closure of the IFR class under formation of coherent systems. IEEE Trans. Reliab. 34, 69-72.

[32] Samaniego, F. J., Balakrishnan, N. and Navarro, J. (2009). Dynamic signatures and their use in comparing the reliability of new and used systems. Naval Res. Logistics 56, 577-591.

[33] Shaked, M. and Shanthikumar, J. G. (2007). Stochastic Orders. Springer, New York.

[34] Sillitto, G. P. (1964). Some relations between expectations of order statistics in samples of different sizes. Biometrika 51, 259-262.

[35] ZarezadeH, S. AND Asadi, M. (2013). Network reliability modeling under stochastic process of component failures. IEEE Trans. Reliab. 62, 917-929.

[36] Zarezadeh, S., Asadi, M. and Balakrishnan, N. (2014). Dynamic network reliability modeling under nonhomogeneous Poisson processes. Europ. J. Operat. Res. 232, 561-571.

[37] Zhang, Z. (2010). Mixture representations of inactivity times of conditional coherent systems and their applications. J. Appl. Prob. 47, 876-885.

[38] Zuo, M. J. AND Tian, Z. (2006). Performance evaluation of generalized multi-state $k$-out-of- $n$ systems. IEEE Trans. Reliab. 55, 319-327. 